\title{
Mobile ICT prototype in public health awareness
}

\author{
Dr. C. de Silva \\ Dr. R. Hewapathirana \\ Dr. P. Ranatunga \\ Dr. S. de Zoysa \\ Postgraduate Institute of Medicine, University of Colombo, Sri Lanka. \\ Contact e-mail address: chamaine.1k@gmail.com
}

eHealth Sri Lanka 2010,1(suppl.1):S6

DOI: http://dx.doi.org/10.4038/sljbmi.v1i0.3538

Only the Abstract is available

\begin{abstract}
Public health awareness is a key requirement in a country. In order to render efficient and effective healthcare, the speed of delivering health messages is vital. Various methodologies are adopted in disseminating health messages worldwide. One effective means is mobile alerts. Since the usage of mobiles phones across the island is so vast, a system was developed to divulge valuable health messages/tips via location based Short Message Service (SMS). Proposed system will enhance two major aspects of health awareness namely regular updates of real time disease burden and health tips of certain diseases which can lead to a crisis situation if uncontrolled. For example; Dengue and Location based disease related health tips notification. Disease pattern differs from one area to the other. Examples: Leptospirosis which is more prevalent in Kurunegala, Rathnapura districts; whereas Dysentery is more common in Batticaloa. Hence if the public get an alert about such a diseases and how to protect themselves upon entering the area it would of utmost use for both the public as well as the preventive health staff. This system generated SMS alerts will comprise of relevant information of public interest of the concerned disease to that area. The preventive measures about chemoprophylaxis if relevant, is the closest hospital in case of emergency. Furthermore the subscriber will get a choice to plan his/her trip ahead. Subscriber is expected to send a SMS with the destinations to the system in turn he/she will get the relevant health tips well ahead. The credible data source on diseases is the Weekly Epidemiological Report (WER).The database will be continuously updated in parallel with updating of WER. This system will be a promising tool in enhancing public health awareness to ensure quality healthcare.
\end{abstract}

Keywords - Public health, delivering health messages, chemoprophylaxis 